\title{
Design of Instant Communication Software based on Socket
}

\author{
Lina Gao \\ College of Information Science and Technology, Bohai University, Jinzhou, 121013, China \\ 21481199@qq.com
}

Keywords: Socket; Instant communication; software design; requirement analysis; functional design; system architecture

\begin{abstract}
Instant communication refers to the Internet for real-time communication system services, allow multiplayer real-time text message, documentation, such as voice and video information flow. In this paper, based on the Socket technology research, provide solution for enterprise instant communication software design. On the basis of the Socket technology, the main research content of the four aspects: first, a brief requirements analysis, including the system analysis of communication process and function; The second is, the system function design, design the function of the client and the server respectively; The third it is, the system architecture design, separate network communication layer and application system of hierarchical architecture; Four is, class design the server side, including CDataSocket classes and class CListenSocket. Instant messenger, relative to the individual enterprise instant communication more emphasis on safety, practicality, stability and expansibility.
\end{abstract}

\section{Introduction}

Instant communication is refers to the service system of real-time communication on the Internet, allow people to use instant messaging software real-time text message, documentation, such as voice and video information flow. With the improving of the software technology and the improvement of the related network infrastructure, the function of the instant communication software is also increasingly rich, in addition to the basic communication function, gradually integrated with emails, blogs, music, television, games and search and other functions, these functions have also prompted instant communication is no longer a simple chat tool, has been successful with communication, entertainment, business office and customer service features such as the integration of information platform.

Instant communication tools, real-time interaction and rates is low wait for an advantage, gradually popular with users, has become an integral part of the network life. Netizens communicating through instant communication, making new friends, entertainment time, implement different text, files, voice, videos, real-time communication exchange. At the same time, people also realize that instant messaging tools can bring high productivity. As the most frequently used network software, instant messaging is breakthrough the limit as a technical tool, is considered to be the new symbol of modern way of communication. In this paper, based on the Socket technology research, for the development of new instant messaging software provides design scheme.

\section{Socket Technology}

The two programs on the network through a two-way communication link for data exchange, one end of the connection is called a Socket. To distinguish different application processes and connection, the operating system for the application and the TC/IP protocol interaction provides a Socket interface, to distinguish the different network communication and the connection between the application processes. Generate a socket, there should be three parameters: communication destination IP address, the transport layer protocol and port number. Socket's original intention is "outlet", combine the three parameters, and a "Socket" Socket binding, can application layer and transport layer through a Socket interface, distinguish from different application process or network 
connection communication, realize the data transmission of concurrent service.

To communicate via the Internet, at least need a pair of sockets, a run on the client side, called ClientSocket; And another run on the server side, called ServerSocket. Connect according to start the way and the local socket to connect the target, the socket can be divided into the connection between the process servers monitoring, client requests and connect to confirm three steps. Monitoring, among them, the server is in a state of waiting for the connection, the real-time monitoring network state; Client request, the client socket connection request is put forward, is to connect the server socket; Connection, the server socket listening to client socket connection request, send the description of the server socket to the client. The Socket is a bridge between the client and the server, as shown in Fig. 1.

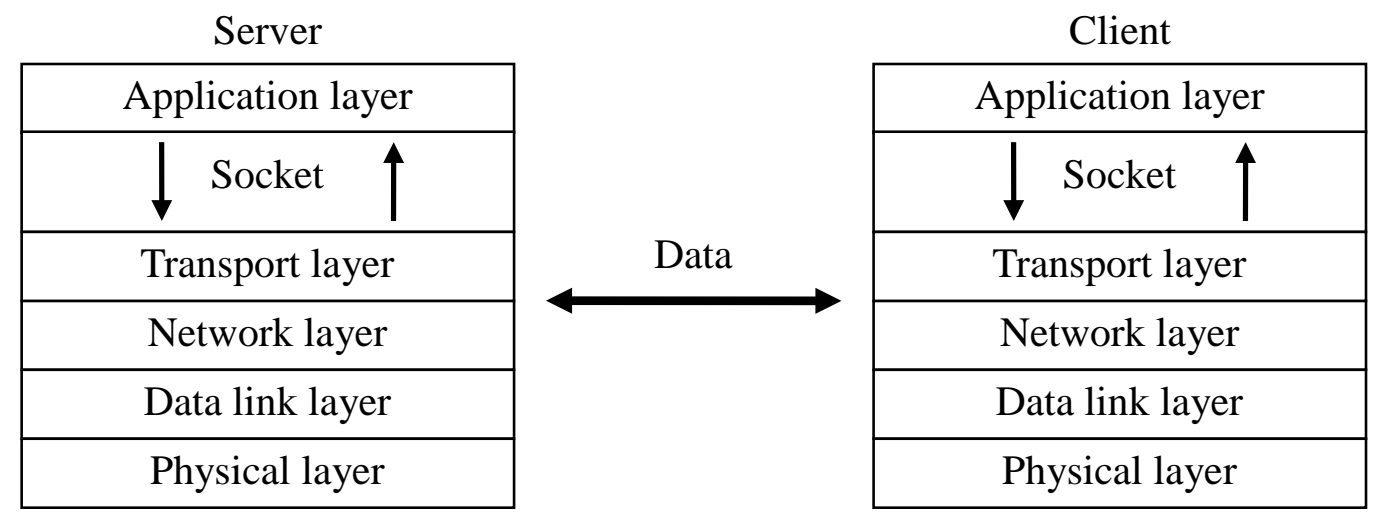

Fig. 1. Communication between client and server

\section{Briefly Requirement Analysis}

Systems analysts to identify the needs of users, comprehensive and modeling, clear user requirements of vagueness, ambiguity and inconsistencies, and data analysis system, this software logic model is set up for the original problem and target. Analysis personnel to the understanding of the original problem with software development experience, in order to find what requirement is due to the user's partial or short-term behavior caused by the unreasonable requirement, which is the user has not been proposed but the potential demand of real value. Demand analysis is important, because have the function of the decision-making, directivity, strategic, has a pivotal position in the software development process. Requirement analysis is an effective way to get users demand, demand analysis is the key factor that decides the success of the project, the requirements analysis is the bridge of system analysis and software design, requirement analysis is an important stage of software quality control. Demand analysis, should pay attention to all information and the demand are standing in the user's point of view, try to avoid the analyst's subjective imagination, try to analysis schedule submitted to the user. On the premise of not direct guidance, let the user for inspection and evaluation, ensures the accuracy of demand analysis.

In communication system based on Socket, the client and the server keep connection. This way to connect is in different way comparing general web browsing. When using a browser to access the server, first made by the client network communication protocol, then the server response processing, and returns the processing result, the request and response is causal relationship between before and after the point to point. And based on Socket communication system, the client communication information at the same time, the client is also in many other communication information server, therefore, requesting and response without corresponding relations between before and after, are two separate processes, respectively. Server could send messages at any time to the client, the client and the server once a connection is established, and must be able to have the server in the future when sending information to find this connection. Instant communication system structure is shown in Fig. 2.

Fig. 2 shows that instant messaging program is divided into client and server two parts. Client mainly realize the following functions: to online members to send messages and receive messages, 
update the member list, when other members to join, the client update member list, members login; Server mainly realize the following functions: to authenticate member login information, notify the client update member state, to accept the request, deliver the message.

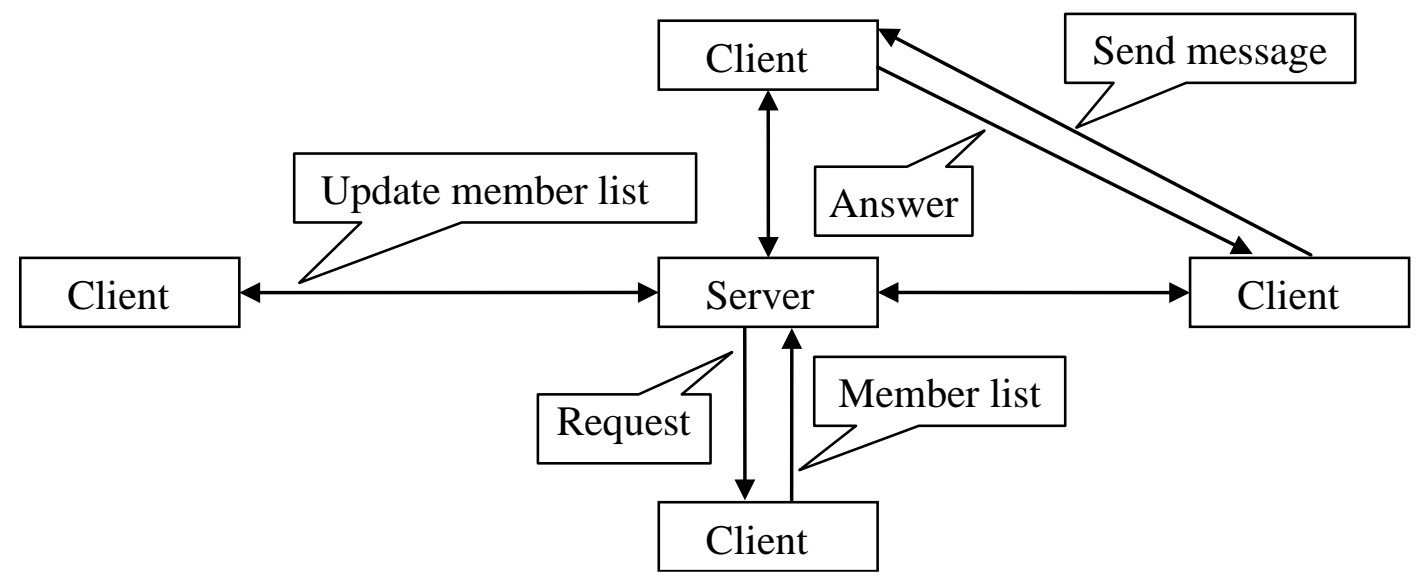

Fig. 2. Instant messaging system structure

\section{System Function Design}

Function Design using modular design method. Modular design should follow the abstraction, incremental refinement, the principle of modularity and information hiding, etc. At the highest levels of abstraction, you can use the problems of environment language description method; Stepwise refinement is a kind of top-down design strategy, the software architecture, according to the top-down approach, details and data details the process of all levels of refinement step by step, until can statements in a programming language to realize; Modularity means "software is made up of several discrete parts of discrete degree, namely software modular degree". Information hiding, is refers to the implementation details of each module is hidden to other modules. Instant communication software function design is based on Socket as shown in Fig. 3.

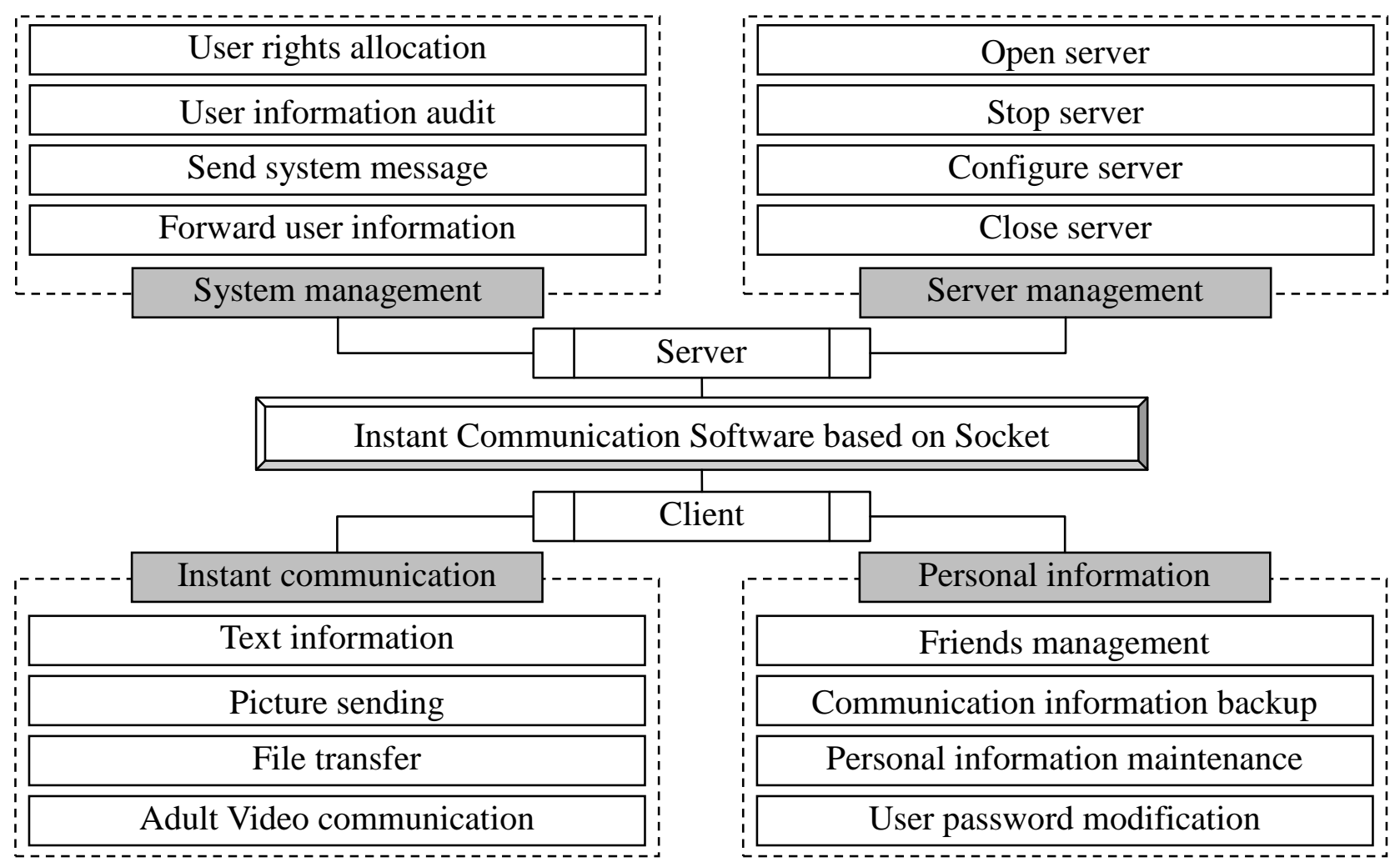

Fig. 3. Software function design 


\section{System Architecture Design}

Software architecture is a sketch of the system. The software architecture description of object is directly constituted a system of abstract components. The connection between the various components are clear and relatively detailed describe the communication between the components. In the implementation phase, these abstract components are divided into actual components, such as a specific class or object. In the field of object oriented, the connection between the components usually use interface to implement. Software architecture is the basis of the constructing practice of computer software, system design to meet different customer needs.

System should have the characteristics of reusable and can be extended, the network communication layer and application system. Based on the network communication layer reusable, can achieve a variety of other real-time application system. Admiral system in the architectural design of four levels, as shown in figure 4.Bottom is the Socket communication layer, is responsible for the data exchange between the client and the server, through the top interface layer and application layer maintain real-time data communication. The Socket of the underlying design is divided into two parts: protocol design and connection handle design; CSocket purpose is to provide the ground floor and the role of the media in the application layer mediation, but can't be too complicated, prevent data transmission time delay.

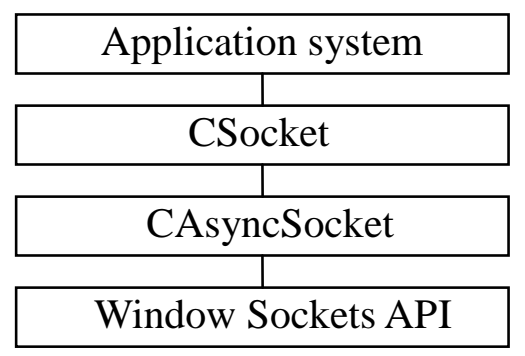

Fig. 4. System architecture

\section{Design on Main Classes in Server}

Classes and objects is the most basic concepts of object-oriented programming technique is the entity in the real world or thinking world reflects in the computer, the data and the data operations on packaging together. Object is a class type variable. Object class is abstract, and the object of the class is specific instances. Class is abstract, which do not take up memory object is concrete, take up the storage space. Class object is used to create a blueprint, is a definition included in a specific type of object template variables and the method of software. The server class mainly includes the following two:

(1) CDataSocket classes, create packets, according to the packet types receive, check the client's login information, login here can be related to verification, receive messages sent by the client log out, and some other related news, etc. In the class member functions include: the CDataSocket (), the constructor; the CDataSocket (), and the destructor; OnClose (int nErrorCode), close the asynchronous Socket; OnReceive (int nErrorCode) receives the message/parsing packet types; OnReceiveLogin (), parse the login message; OnReceiveLogout (), access to logout the user's messages; OnReceiveMessage () and displays a message.

(2) CListenSocket class, derived from MFC CSocket class, to accept the client's connection request, class overrides the OnAccept CAsyncSocket class () function. When the client connect to the server, OnAccept () function is invoked. CServInfoDlg: use and set the address of the server and fracture. CServerApp: used to generate the only instance. In the class member functions include: CListenSocket (), the constructor; CListenSocket (), and the destructor; VoidOnAccept (intnErrorCode), accept the connection request.

Building function and destructors are synonymous with class, the constructor, is a special kind of method, mainly used to initialize object when creating objects, namely member variable an initial value for the object, always use with new operator in the statement of object creation. Special a 
class can have multiple constructors, according to the different number of parameters or parameter to distinguish between different types, namely constructor overload; Destructor, in contrast to the constructor, when the object to end its life cycle (such as object's function has been called), the system automatically perform the destructor. Destructors are often used to do the work of "clean up" (for example, in establishing objects in the new opened up a piece of memory space, should be in front of the exit in the destructor in the delete release).

\section{Conclusion}

Most common network real-time communication system for the entire group of Internet users, in addition to the communication, is bound to a series of rich applications and services, such as social networking, games, music and portal, etc., to provide users with rich experience and high level of integration of network application. But demand for IM product enterprises and some fields with existing products, provided by the application provider does not need complex variety of entertainment applications and a free and open registration environment, pay more attention to the account management, application of concise and environmental safety, more and more applied in the local area network (LAN) or virtual local area network environment, the domestic demand for such products. Research of this paper is the field application and design, has a broad market prospect.

\section{References}

[1] M. J. Wei, M. F. Liu, "Instant Communication System Based on Local Area Network," Computer Knowledge and Technology, vol. 12, no. 5, pp. 39-40, 2016.

[2] J. Z. Wan, L. S. Zhong, X. Deng, J. Wen, "Design of local area network instant communication system under.NET framework," Fujian Computer, vol. 29, no. 9, pp. 30-31, 2013.

[3] Blog "Simple understanding Socket," http://www.cnblogs.com/dolphinX/p/3460545.html, 2016-9-15.

[4] Xiaoweige207 column, "Socket communications profile," http://blog.csdn.net/xiaoweige207/article/details/6211577, 2016-9-15.

[5] J. L. Sun, "Design and Implementation of a Instant Message System for LAN," Master's degree of Huazhong University of Science and Technology, 2011.

[6] N. Liu, "Design and implementation of a real time communication system," Master's degree of Yunnan University, 2012. 